\title{
Effect of Peptide Receptor Radionuclide Therapy on Somatostatin Receptor Status and Glucose Metabolism in Neuroendocrine Tumors: Intraindividual Comparison of Ga-68 DOTANOC PET/CT and F-18 FDG PET/CT
}

\author{
Sowon Oh, ${ }^{1}$ Vikas Prasad, ${ }^{2}$ Dong Soo Lee, ${ }^{1}$ and R. P. Baum ${ }^{2}$ \\ ${ }^{1}$ Department of Nuclear Medicine, College of Medicine, Seoul National University, Seoul 151-742, Republic of Korea \\ ${ }^{2}$ Department of Nuclear Medicine, Center for PET/CT, Zentralklinik Bad Berka, Robert Koch Allee 9, 99437 Bad Berka, Germany
}

Correspondence should be addressed to R. P. Baum, richard.baum@zentralklinik.de

Received 25 May 2011; Revised 10 August 2011; Accepted 18 August 2011

Academic Editor: Xiaoyuan Chen

Copyright (C) 2011 Sowon Oh et al. This is an open access article distributed under the Creative Commons Attribution License, which permits unrestricted use, distribution, and reproduction in any medium, provided the original work is properly cited.

\begin{abstract}
The heterogeneous nature of the neuroendocrine tumors (NET) makes it challenging to find one uniformly applicable management protocol which is especially true for diagnosis. The discovery of the overexpression of somatostatin receptors (SMS-R) on neuroendocrine tumor cells lead to the generalized and rapid acceptance of radiolabeled somatostatin receptor analogs for staging and restaging of NET as well as for Peptide Receptor Radionuclide Therapy (PRRNT) using Y-90 and Lu177 DOTATATE/DOTATOC. In this present work we tried to look in to the effect of PRRNT on the glucose metabolism assessed by F-18 FDG PET/CT and SMS-R density assessed by Ga-68 DOTANOC PET/CT. We observed a complex relationship between the somatostatin receptor expression and glucose metabolism with only 56\% (77/138) of the lesions showing match, while the others show mismatch between the receptor status and metabolism. The match between receptor expression and glucose metabolism increases with the grade of NET. In grade 3 NET, there is a concurrence between the changes in glucose metabolism and somatostatin receptor expression. PRRNT was found to be more effective in lesions with higher receptor expression.
\end{abstract}

\section{Introduction}

Neuroendocrine tumours (NETs) are a rare but very heterogeneous group of neoplasms $[1,2]$. NETs are generally slow-growing tumors having a relatively good prognosis. Although many of NETs are clinically silent, presenting with symptoms of mass effects or distant metastases [13], they often lead to debilitating symptoms like diarrhea and flush, which deteriorates patients' quality of life [4]. Surgery is the only treatment having curative intent, while cold somatostatin analogues are primarily used for reducing the symptoms $[2,4,5]$. However, the inhibition of secretion of the peptides responsible for symptoms is often transient as the median duration of improvement of carcinoid symptoms by octreotide is known to be over 12 months [6]. Furthermore, somatostatin analogues seldom have antitumor effect, specifically if more than $10 \%$ of the liver is involved with tumor [7-11].
Somatostatin receptor scintigraphy (SRS) is still considered as the gold standard for staging of NET. However, recent studies have clearly shown the superiority of Ga68 somatostatin receptor PET/CT over SRS [12-14]. High tumor uptake on SRS is reported to be associated with better tumor response to PRRNT $[15,16]$ and that is the reason why tumor uptake on SRS has been suggested as a new prognostic factor in well-differentiated endocrine tumors [17, 18]. ${ }^{68} \mathrm{Ga}$ DOTANOC has been shown to have a very good binding affinity to somatostatin receptor subtypes 2 and 3 and higher binding affinity to somatostatin receptor subtype 5 as compared to DOTATATE and DOTATOC [19]. On the other hand, ${ }^{18} \mathrm{~F}$-fluorodeoxyglucose (FDG) has only limited value in good differentiated NET and is recommended only if SRS is negative especially in poorly differentiated NET $[20,21]$. Additionally, ${ }^{68} \mathrm{Ga}$ DOTATATE PET/CT has been shown to be a useful novel imaging modality for NETs and was found to be superior to ${ }^{18} \mathrm{~F}-\mathrm{FDG}$ for imaging well-differentiated 
TABLE 1: Demographics and clinical characteristics.

\begin{tabular}{lc}
\hline Characteristics & No. \\
\hline Age & $58.7 \pm 12.2$ \\
$\quad$ Mean \pm SD & \\
Sex, $n(\%)$ & $16(64 \%)$ \\
$\quad$ Male & $9(36 \%)$ \\
Female & \\
Pathology & 18 \\
GEP-NET & 2 \\
CUP & 4 \\
Carcinoid & 1 \\
Pheochromocytoma & \\
Number of PRRT & 17 \\
1 & 8 \\
up to 3 &
\end{tabular}

GEP-NET: gastroenteropancreatic neuroendocrine tumor.

NET in previous study. However, functional imaging with both ${ }^{68} \mathrm{Ga}$ DOTATATE and ${ }^{18} \mathrm{~F}$-FDG has shown to address different biological properties of the neuroendocrine tumor lesions in patients planned for ${ }^{90}$ YDOTATOC PET/CT [22] and has been proposed as for comprehensive tumor assessment in intermediate- and high-grade tumors [23]. In our study, an intraindividual comparison was made between the somatostatin receptor status using ${ }^{68} \mathrm{Ga}$ DOTANOC $\mathrm{PET} / \mathrm{CT}$ and glucose metabolism using ${ }^{18} \mathrm{~F}$ FDG PET/CT after PRRNT.

\section{Materials and Methods}

2.1. Patient Population. Overall, 25 patients with progressive, metastasized neuroendocrine tumor were included. All the patients were imaged using FDG-PET/CT and ${ }^{68} \mathrm{Ga}$ DOTANOC PET/CT.

The average time interval between the two different PET/CTs was 3 days (mean age of $58.7 \pm 12.2$ years; female to male ratio, 9:16). For precise lesion-by-lesion comparison and to avoid any partial volume effect, lesions larger than $2 \mathrm{~cm}$ in diameter were measured. The metastatic lesions that were too confluent or conglomerated to be separately analyzed were excluded from the study. Detailed demographic data are shown in Table 1.

\subsection{Radiopharmaceutical Preparation}

${ }^{68} \mathrm{Ga}$ DOTANOC Labeling. To perform receptor PET/CT, ${ }^{68} \mathrm{Ga}$ was eluted from ${ }^{68} \mathrm{Ge} /{ }^{68} \mathrm{Ga}$ generator (obtained from Eckert and Ziegler, Berlin, Germany), and the labeling process and associated quality control were performed according to the technique mentioned in a previous publication [24, $25]$.

${ }^{90} Y$ DOTATATE and ${ }^{177}$ Lu DOTATATE. Both ${ }^{90} \mathrm{Y}$ DOTATATE and ${ }^{177} \mathrm{Lu}$ DOTATATE were prepared in house, in a class 10 facility, under strict GMP protocol. The radiochemical purity (checked using HPLC) of the final preparation was always $>99 \%$. The total amount of peptide administered in each cycle of ${ }^{90} \mathrm{Y}$ DOTATATE and ${ }^{177} \mathrm{Lu}$ DOTATATE ranged from 75 to $250 \mu \mathrm{g}$ and from 160 to $500 \mu \mathrm{g}$ respectively.

2.3. Imaging Protocols. A dual-modality PET/CT tomography (Biograph duo; Siemens Medical Solutions) was used, which consists of a PET system with a full-ring lutetium oxyorthosilicate (LSO) and a CT component corresponding to a Somatom Emotion Duo (Siemens Medical Solutions), a 2-row spiral CT system with a maximum continuous scan time of $100 \mathrm{~s}$ and a maximum rotation speed of $75 \mathrm{rpm}$. Water-equivalent iodine containing contrast agent was given intravenously at 60 minutes before starting the ${ }^{68} \mathrm{Ga}$ DOTANOC PET/CT. This dispersion is routinely used in PET/CT without known adverse side effects on the accumulation of ${ }^{68} \mathrm{Ga}$ DOTANOC. For ${ }^{18} \mathrm{~F}$-FDG scan, fasting for more than 12 hours was additionally requested. In addition, urine voiding was asked to all patients immediately before the PET/CT. The image acquisition started 60 minutes after administration of approximately $120 \mathrm{MBq}$ of ${ }^{68} \mathrm{Ga}$ DOTANOC and $250 \mathrm{MBq}{ }^{18} \mathrm{~F}$-FDG, while the patients were positioned head first supine with the arms raised in accordance with a standard CT practice and following the EANM guidelines [26, 27]. The acquisition parameters were kept strictly the same for pre- and post-PRRNT PET/CT imaging.

A topogram was acquired over $1.024 \mathrm{~mm}$ axially, and coaxial whole-body imaging ranges were defined on the topogram from the skull to the upper thighs (7-8 PET bed positions, or $90-100 \mathrm{~cm}$, depending on the size of the patient). Intravenous radioiodine contrast media of 100 milileters was injected by an automated injection pump after checking the topogam, and contrast-enhanced CT images were acquired during venous phase of the contrast from the level of the skull to the thighs with a $30 \mathrm{~s}$ delay CT in spiral mode using a continuous acquisition at $130 \mathrm{kVp}, 115 \mathrm{mAs}$, $4 \mathrm{~mm}$ collimation, $5 \mathrm{~mm}$ slice width, a table feed of $8 \mathrm{~mm}$ per rotation at $0.8 \mathrm{~s}$ rotation time, and $2.4 \mathrm{~mm}$ slice spacing. For the CT image acquisition, a limited breath hold protocol was required to the patients. Then PET emission scan was obtained in 3-dimensional mode at each bed position for 1-2 minutes (depending upon the weight and height of the patient) for the same coverage with the CT images, which started in the caudocranial direction after the patients moving automatically to the PET toward the rear of the gantry. As a result, a total emission scan time did not exceed 24 minutes, and a total PET/CT examination took about 30 minutes including patient positioning, CT, and PET imaging.

2.4. Therapy Protocol. The legal requirements were met in accomplishing the study (including ethical and local radiation protection regulations). The study was performed according to guidelines, approved by the local ethical committee at the Zentralklinik Bad Berka and in accordance with German regulations (as published by the Federal Office for Radiation Protection, BfS) concerning radiation safety. Written informed consent was obtained from all patients 
before therapy. All patients, who were enrolled in this study, presented with progressive neuroendocrine tumors after having exhausted all conventional therapeutic options. Among other factors (Table 1), the degree of somatostatin receptor expression (maximum Standardized Uptake Value $\mathrm{SUV}_{\max }$ ) as determined by Ga-68 somatostatin receptor PET/CT $[28,29]$, number and size of metastases, pretherapy renal function, and hematological profile was primarily taken into consideration before estimation of the dose to be administered, thereby individualizing the patients' therapy.

On an average, each patient received $3.0 \mathrm{GBq}{ }^{90} \mathrm{Y}$ DOTATATE and/or $7.5 \mathrm{GBq}{ }^{177} \mathrm{Lu}$ DOTATATE per cycle. In total, 17 patients were treated with only one cycle of PRRNT, while the remaining 8 patients received upto three cycles.

2.5. Renal Protection. For kidney protection, every patient was coinfused with $2000 \mathrm{~mL}$ of a renoprotective amino acid mixture of 5\% Lysine HCL and 10\% L-Arginine HCL in $250 \mathrm{~mL} \mathrm{NaCl}$ at $\mathrm{pH} 7.4$ and osmolarity of $400 \mathrm{mosmol} / \mathrm{L}$. This infusion was started 30 minutes prior to the administration of the therapeutic dose and continued for 4 hours. The radiopharmaceutical was coadministered over 10-15 minutes by using a second infusion pump system [30]. Each patient was well hydrated by ensuring an intake of at least 1 litre of mineral water before therapy and 2-3 litres thereafter. In case there was evidence of renal obstruction (physiologic or pathological), i.v. injection of $20-40 \mathrm{mg}$ of furosemide in 1.5-2 litre of deltajonin was given over $2-4$ hours after therapy.

2.6. Data Reconstruction. For attenuation correction of the PET emission images, the CT transmission data were used. The PET images were reconstructed iteratively using an attenuation-weighted ordered-subsets expectation maximization algorithm with 2 iterations and 8 subsets on $128 \times$ 128 matrices and with a 5-minute Gaussian postreconstruction filtering.

2.7. Image Evaluation and Analysis. For the analysis of $\mathrm{PET/CT}$, two different imaging viewing systems were used by an experienced radiologist and two experienced nuclear medicine physicians, respectively; a Syngo viewer for the image analysis of CT and an E. soft workstation (Siemens Medical Solutions) for the PET/CT image assessment. At first, the maximum intensity projection (MIP) images were visually examined in varying scales, and then each single transverse slice was looked over from the skull base to the midthighs in combination with the corresponding CT image and the fused image slice. Each lesion showing a focal abnormal tracer uptake was recorded by a slice number and anatomic localization, and any lesion with intensity greater than background which could not be explained by physiological activity was considered to be indicative of tumor tissue. For semiquantitative analysis of the lesions, a region of interest (ROI) automatically drown around each lesion maximum standardized uptake value $S_{U V} V_{\max }$ was evaluated. Up to a maximum of five lesions per organ and 10 lesions in total were chosen in $\mathrm{SUV}_{\max }$ order to avoid bias of statistical counting. Changes in the size on CT $(\Delta \mathrm{CT})$, $\mathrm{SUV}_{\max }$ of FDG $(\triangle \mathrm{FDG})$, and $\mathrm{SUV}_{\max }$ of ${ }^{68} \mathrm{Ga}$ DOTANOC $(\triangle \mathrm{SMS}-\mathrm{R})$ were compared with each other.

Additionally, all the lesions were regrouped based upon the tumor location-primary sites, liver, lymph nodes, and bones.

2.8. Statistical Analysis. $\mathrm{SUV}_{\max }$ of each lesion prior to and after PRRNT were compared with Student's $t$-test. In case that the total number of cases was nonparametric, Wilcoxon signed-rank test and Mann-Whitney $U$ test were adopted. Analysis of variance test was done to compare means of two groups. A value of $P<0.05$ was considered significant. All statistical analysis was carried out using MedCalc statistical software (version 9.3.2; MedCalc, Mariakerke, Belgium).

\section{Results}

3.1. Analysis Based on the Uptake Pattern of Two PET/CTs. In 25 patients, a total of 133 discrete lesions were identified on either ${ }^{68} \mathrm{Ga}$ DOTANOC (SMS) PET/CT or ${ }^{18} \mathrm{~F}$ FDG (FDG) PET/CT. Most lesions $(n=126,94.7 \%)$ were positive on SMS PET/CT, but $61.9 \%(n=78)$ was positive on FDG PET/CT at the same time. The remaining lesions $(n=7,5.3 \%)$ were only detectable by FDG PET/CT and were negative on SMS PET/CT. The lesions were subclassified as follows: both SMS as well as FDG positive (group A: $58.6 \%, n=78$ ), only SMS positive (group B: $n=38,28.6 \%$ ), or FDG positive (group C: $n=7,5.3 \%$ ). The PET/CT findings of the three groups are shown in Figure 1.

In patients where Ki-67 was available $(n=10), 6$ had Ki-67 >20\% (grade 3 NET G3), 2 had Ki-67 between 3 and 20 (grade 2 NET G2), and 2 had Ki-67 <2\% (grade 1 NET, G1). In this subgroup of patients, FDG could pick up $67 \%$ of the receptor-positive lesions (40/61); in G3 NET, FDG PET could pick up $90 \%$ of the lesions (36/39). There was a significant correlation between the $\triangle F D G$ and $\triangle$ SMS-R. There was significant correlation between ( 32 measurable lesions on CT also positive on FDG PET and SMS-R PET) the $\triangle \mathrm{FDG}$ and $\triangle \mathrm{CT}(P=0.027)$, between $\triangle \mathrm{FDG}$ and $\triangle \mathrm{SMS}$ $\mathrm{R}(0.007)$, but no significant change between the $\Delta$ SMS- $R$ and $\Delta \mathrm{CT}$.

In group $\mathrm{A}$, the $\mathrm{SUV}_{\max }$ of SMS $(23.0 \pm 13.6$ versus 17.3 $\pm 9.4, P<0.0001$ ) (see Table 2 and Figure 2 ) as well as the $\operatorname{SUV}_{\max }$ of FDG $(9.0 \pm 4.3$ versus $6.6 \pm 4.2, P<0.0001)$ decreased significantly after PRRNT. The SUV $\mathrm{max}_{\max }$ of SMS also decreased in group B $(14.7 \pm 9.1$ versus $11.5 \pm 8.8, P$ $<0.0001)$, but the SUV ${ }_{\max }$ of FDG in group C $(12.6 \pm 5.0$ versus 16.9 $\pm 8.0, P=0.0469)$ increased after PRRNT. As for the radiotracer uptake pattern, the baseline $S_{U V} V_{\max }$ of SMS in group A was significantly higher than that of group B (23.0 \pm 13.6 versus $14.7 \pm 9.1, P=0.0003$ ). On the other hand, the baseline SUV max $_{\max }$ of FDG in group A tended to be lower than in group $C(9.0 \pm 4.3$ versus $12.6 \pm 5.0)$ though it failed to reach a statistical significance.

3.2. Analysis Based on the Tumor Location. The lesions were regrouped based on the location of the tumor-primary site 


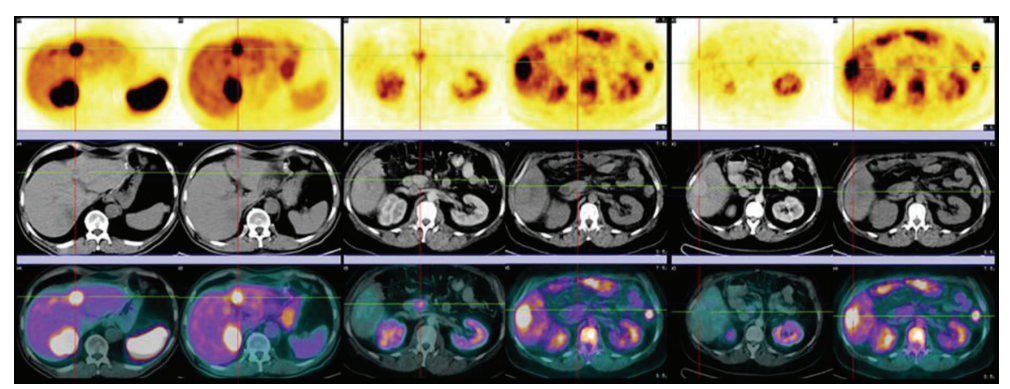

(a)

(b)

(c)

FIGURE 1: Grouping based on the uptake of two PET/CTs. (a) Group A: a metastatic liver lesion presented both SMS (on the left) and FDG uptake (on the right). (b) Group B: a primary tumor in the pancreas head presented SMS uptake (on the left), FDG PET/CT images on the right showed no uptake in the primary. (c) Group C: a metastatic liver lesion presented FDG uptake (on the right) but was negative for somatostatin receptor expression (on the left).

TABLE 2: Response to PRRNT: comparison of SMS-R and FDG PET/CT.

\begin{tabular}{|c|c|c|c|c|c|c|}
\hline \multirow{2}{*}{ Group } & \multirow{2}{*}{$\mathrm{PET} / \mathrm{CT}$} & \multirow{2}{*}{ Response } & \multicolumn{2}{|c|}{ Before PRRT } & \multicolumn{2}{|c|}{ After PRRT } \\
\hline & & & $N$ & $\mathrm{SUV}_{\text {max }}$ & $N$ & $\mathrm{SUV}_{\max }^{\dagger}$ \\
\hline \multirow{8}{*}{ Group A } & \multirow{5}{*}{ SMS } & $\mathrm{CR}+\mathrm{PR}$ & 44 & $27.4 \pm 14.9$ & 41 & $16.3 \pm 9.9^{\dagger}$ \\
\hline & & $\mathrm{SD}$ & 24 & $19.1 \pm 9.9$ & 24 & $18.0 \pm 9.5^{\dagger}$ \\
\hline & & $\mathrm{PD}$ & 10 & $12.9 \pm 5.6$ & 10 & $19.9 \pm 7.5^{*}$ \\
\hline & & Total & 78 & $23.0 \pm 13.6$ & 75 & $17.3 \pm 9.4^{\dagger}$ \\
\hline & & $\mathrm{CR}+\mathrm{PR}$ & 44 & $9.4 \pm 4.4$ & 37 & $6.5 \pm 4.1^{\dagger}$ \\
\hline & \multirow{3}{*}{ FDG } & $\mathrm{SD}$ & 24 & $8.2 \pm 3.7$ & 20 & $6.3 \pm 3.2^{*}$ \\
\hline & & $\mathrm{PD}$ & 10 & $8.9 \pm 5.1$ & 7 & $7.1 \pm 6.6$ \\
\hline & & Total & 78 & $9.0 \pm 4.3$ & 64 & $6.6 \pm 4.2$ \\
\hline \multirow{4}{*}{ Group B } & \multirow{4}{*}{ SMS } & $\mathrm{CR}+\mathrm{PR}$ & 25 & $12.9 \pm 9.3$ & 15 & $6.7 \pm 6.5^{\dagger}$ \\
\hline & & $\mathrm{SD}$ & 20 & $17.4 \pm 8.9$ & 20 & $16.5 \pm 8.4^{*}$ \\
\hline & & $\mathrm{PD}$ & 3 & $12.0 \pm 5.2$ & 3 & $18.4 \pm 5.8$ \\
\hline & & Total & 48 & $14.7 \pm 9.1$ & 38 & $11.5 \pm 8.8$ \\
\hline Group C & FDG & Total & 7 & $12.6 \pm 5.0$ & 7 & $16.9 \pm 8.0^{*}$ \\
\hline
\end{tabular}

${ }^{\dagger}: P<0.0001,{ }^{*}: P<0.05$.

( $n=19,14.3 \%)$, lymph nodes metastases $(n=26,19.5 \%)$, liver metastases $(n=71,53.4 \%)$, and bone metastases $(n$ $=17,12.8 \%)$. The lesions were further classified according to the receptor expression and FDG uptake; matched group contained lesions showing somatostatin receptor expression and FDG uptake, and the mismatched group contained lesions which were either somatostatin receptor-positive lesion or FDG positive. In the liver, FDG PET/CT found additional lesions $(n=7)$ which did not accumulate Ga-68 DOTANOC. All the other lesions were somatostatin receptor positive.

The pattern of SMS and FDG changes of the tumor in response to PRRNT differed by its location (see Table 3 ). The receptor expression as detected by $\mathrm{SUV}_{\max }$ in the primary tumor $(23.2 \pm 11.0$ versus $17.6 \pm 8.0, P=0.037)$ and lymph nodes $(21.3 \pm 13.6$ versus $15.3 \pm 9.6, P<$ 0.0001) decreased significantly after PRRNT, whereas the corresponding FDG did not show any significant change. In the mismatched groups of the primary tumor and lymph nodes, no significant change was seen in the SMS uptake after the PRRNT application; the baseline $\mathrm{SUV}_{\max }$ were lower than those of matched groups (primary tumor: $14.5 \pm 13.5$ versus $23.2 \pm 11.0, P=0.043$, lymph nodes: $9.5 \pm 5.1$ versus $21.3 \pm 13.6, P=0.018)$. On the contrary, metastatic bone lesions showed a significant decrease of the Ga-68 DOTANOC uptake only in the mismatched group $(7.5 \pm 3.1$ versus $5.1 \pm 3.1, P=0.007)$. The FDG uptake of the bone lesions also decreased significantly $(8.6 \pm 3.2$ versus $3.2 \pm$ $1.6, P=0.007)$. In the matched group, the bone lesions which were also FDG avid did not show any significant decrease on receptor expression on SMS-R PET. In the liver, the changes of each PET/CT were different among the three subgroups. The matched group of the liver showed significant decreases in both SMS uptake $(26.5 \pm 13.9$ versus $19.6 \pm 9.5, P=$ $0.0001)$ and FDG uptake $(8.8 \pm 4.2$ versus $6.2 \pm 3.6, P<$ $0.0001)$. In the mismatched group, the $\mathrm{SUV}_{\max }$ of the liver lesions showing receptor expression decreased after PRRNT $(19.0 \pm 7.4$ versus $14.9 \pm 5.7, P=0.0023)$, whereas the $\mathrm{SUV}_{\max }$ of the hepatic lesions showing only FDG uptake increased $(12.6 \pm 5.0$ versus $16.9 \pm 8.0, P=0.0469)$. 


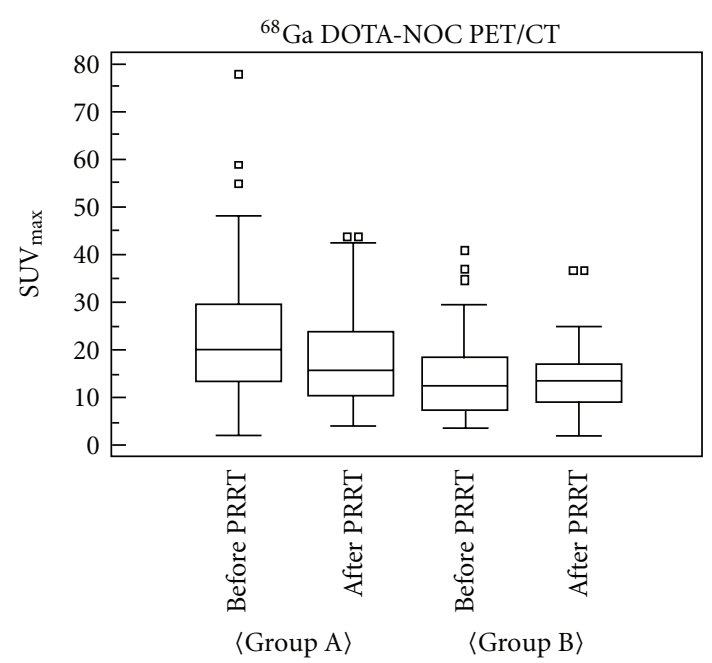

(a)

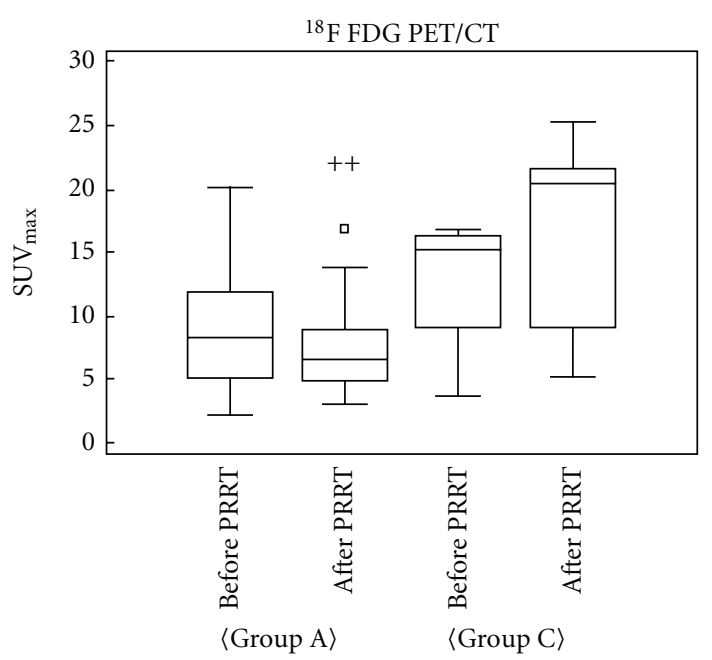

(b)

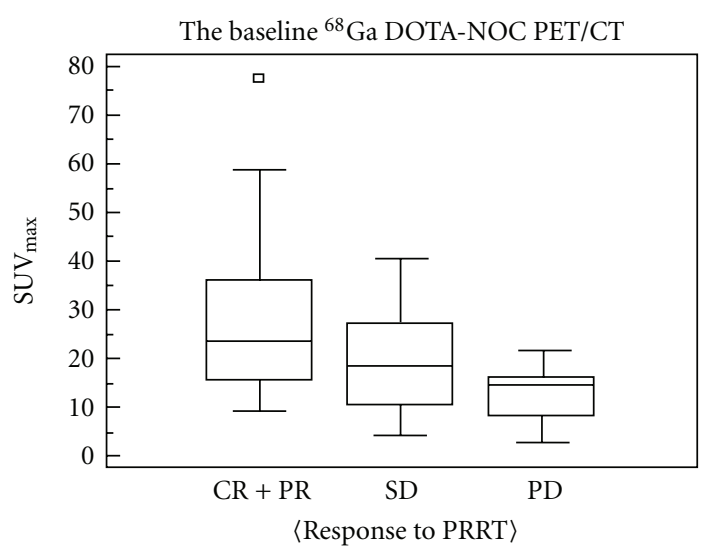

(c)

FIGURE 2: Relationship between SUV $\max$ before and after PRRNT. (a) Changes of SUV on ${ }^{68}$ Ga DOTANOC (SMS) PET/CT in response to PRRNT. The SMS uptake decreased significantly in both group A and group B; baseline $\mathrm{SUV}_{\max }$ of group A was higher than that of group B. (b) SUV changes on ${ }^{18} \mathrm{~F}$ FDG (FDG) PET/CT in response to PRRNT. The FDG uptake decreased significantly in group A, whereas the uptake in group $\mathrm{C}$ increased. The baseline $\mathrm{SUV}_{\max }$ of group $\mathrm{C}$ tended to be higher than that of group A. (c) The relationship between therapy response and baseline $\mathrm{SUV}_{\max }$ of ${ }^{68} \mathrm{Ga}$ DOTANOC PET/CT. The response is positively correlated with the baseline $S U V_{\max }$.

\section{Discussion}

It is known that NETs except benign insulinomas highly express SMS with an incidence of $80-100 \%$, and its density is also predominantly high [31]. In this study, most lesions were well visualized on SMS PET/CT, and more than half of the lesions showed response by means of PRRNT. Also, higher tumor remission rate was correlated with a high-baseline $\mathrm{SUV}_{\max }$ on SMS PET/CT. This finding is well consistent with previous studies $[16,32]$, and PRRNT seems to be quite an effective therapy option for NET patients expressing adequate densities of SMS on the tumors.

To the best of our knowledge, this study has shown for the first time that PRRNT seemed to have an effect on glucose metabolism of NETs showing somatostatin receptor expression. It is well known that the changes in the FDG correlate significantly with tumor response [33]. Because the $\triangle F D G$ showed significant correlation with the $\triangle \mathrm{SMS}$, the changes in $\triangle \mathrm{SMS}$ could be taken as a measure of the response assessment.

This study also showed, as expected, that PRRNT is only effective in lesions showing adequate SMS expression. This was the reason why receptor-negative hypermetabolic lesions progressed after PRRNT.

The main use of FDG PET in diagnosis of NETs depends on the grade of differentiation and/or aggressiveness of NETs. In our patient population with proliferation rate of more than $20 \%$, a very high percentage of receptor-positive lesions were also picked up by FDG PET. Interestingly, nearly all the measured lesions showing FDG uptake, even in patients with high proliferation rate, showed good response to PRRNT on CT as well as on SMS-R PET. Similar results have been observed by Ezziddin et al. where the authors showed that the response to PRRNT is very good even if the proliferation 
TABLE 3: Different response patterns according to tumor location.

\begin{tabular}{|c|c|c|c|c|c|c|}
\hline \multirow{2}{*}{ Location } & \multirow{2}{*}{ Group } & \multirow{2}{*}{$\mathrm{PET} / \mathrm{CT}$} & \multicolumn{2}{|c|}{ Before PRRT } & \multicolumn{2}{|c|}{ After PRRT } \\
\hline & & & $N$ & $\mathrm{SUV}_{\text {max }}$ & $N$ & $\mathrm{SUV}_{\text {max }}$ \\
\hline \multirow{3}{*}{ Primary sites } & \multirow{2}{*}{ Mat } & SMS & 10 & $23.2 \pm 11.0$ & 9 & $17.6 \pm 8.0^{*}$ \\
\hline & & FDG & 10 & $9.0 \pm 4.2$ & 9 & $8.2 \pm 4.2$ \\
\hline & Mis & SMS & 9 & $14.5 \pm 13.5$ & 5 & $12.9 \pm 14.7$ \\
\hline \multirow{3}{*}{$\mathrm{LN}$} & \multirow{2}{*}{ Mat } & SMS & 17 & $21.3 \pm 13.6$ & 17 & $15.3 \pm 9.6^{\dagger}$ \\
\hline & & FDG & 17 & $9.5 \pm 5.1$ & 16 & $8.4 \pm 5.3$ \\
\hline & Mis & SMS & 9 & $10.5 \pm 5.1$ & 5 & $7.5 \pm 7.4$ \\
\hline \multirow{4}{*}{ Liver } & \multirow{2}{*}{ Mat } & SMS & 42 & $26.5 \pm 13.9$ & 39 & $19.6 \pm 9.5^{\dagger}$ \\
\hline & & FDG & 42 & $8.8 \pm 4.2$ & 32 & $6.2 \pm 3.6^{\dagger}$ \\
\hline & \multirow{2}{*}{ Mis } & SMS & 22 & $19.0 \pm 7.4$ & 21 & $14.9 \pm 5.7^{*}$ \\
\hline & & FDG & 7 & $12.6 \pm 5.0$ & 7 & $16.9 \pm 8.0^{*}$ \\
\hline \multirow{3}{*}{ Bone } & \multirow{2}{*}{ Mat } & SMS & 9 & $9.9 \pm 3.5$ & 9 & $9.6 \pm 4.9$ \\
\hline & & FDG & 9 & $8.6 \pm 3.2$ & 7 & $3.2 \pm 1.6^{*}$ \\
\hline & Mis & SMS & 8 & $7.5 \pm 3.1$ & 7 & $5.1 \pm 3.1^{*}$ \\
\hline
\end{tabular}

Abbreviations: Mat: matched, Mis: mismatched, LN: lymph nodes.

${ }^{\dagger}: P<0.0001,{ }^{*}: P<0.05$.

rate is roughly $20 \%$ [34]. This observation supports the use of PRRNT in somatostatin receptor-positive NET. However, there is as of yet no consensus or literature comparing PRRNT with chemotherapy in grade 3 NET.

One of the most interesting observations in this study was the difference in the pattern of response to PRRNT based on the tumor localization. The response to PRRNT was indifferent to the glucose metabolism in the lymph node metastases, liver metastases, and the primary tumor. However, the hypermetabolic bone lesions did not show any significant response to PRRNT. This difference could be related to the different biological properties of tumor cells in bone or bone marrow as compared to those metastasizing to liver or lymph nodes. This observation needs to be validated in further studies.

\section{Conclusion}

There is a complex relationship between the somatostatin receptor expression and glucose metabolism with only $56 \%$ (77/138) of the lesions showing match, while the others show mismatch between the receptor status and metabolism. The match between receptor expression and glucose metabolism increases with the grade of NET. In grade $3 \mathrm{NET}$, there is a concurrence between the changes in glucose metabolism and somatostatin receptor expression. PRRNT is more effective in lesions with higher receptor expression.

\section{References}

[1] M. T. Barakat, K. Meeran, and S. R. Bloom, "Neuroendocrine tumours," Endocrine-Related Cancer, vol. 11, no. 1, pp. 1-18, 2004.

[2] I. M. Modlin, K. Mark, I. Latich, M. N. Zikusoka, and M. D. Shapiro, "Current status of gastrointestinal carcinoids," Gastroenterology, vol. 128, no. 6, pp. 1717-1751, 2005.
[3] G. A. Kaltsas, G. Besser, and A. B. Grossman, "The diagnosis and medical management of advanced neuroendocrine tumors," Endocrine Reviews, vol. 25, no. 3, pp. 458-511, 2004.

[4] K. Oberg and B. Eriksson, "Medical treatment of neuroendocrine gut and pancretic tumors," Acta Oncologica, vol. 28, no. 3, pp. 425-431, 1989.

[5] D. J. Kwekkeboom, E. P. Krenning, R. Lebtahi et al., "ENETS consensus guidelines for the standards of care in neuroendocrine tumors: peptide receptor radionuclide therapy with radiolabeled somatostatin analogs," Neuroendocrinology, vol. 90, no. 2, pp. 220-226, 2009.

[6] L. K. Kvols, C. Moertel, and M. J. O'Connell, “Treatment of the malignant carcinoid syndrome. Evaluation of a long-acting somatostatin analogue," The New England Journal of Medicine, vol. 315, no. 11, pp. 663-666, 1986.

[7] A. Rinke, H. H. Muller, C. Schade-Brittinger et al., "Placebocontrolled, double-blind, prospective, randomized study on the effect of octreotide LAR in the control of tumor growth in patients with metastatic neuroendocrine midgut tumors: a report from the PROMID study group," Journal of Clinical Oncology, vol. 27, no. 28, pp. 4656-4663, 2009.

[8] S. Faiss, U. Pape, M Bohmig et al., "Prospective, randomized, multicenter trial on the antiproliferative effect of lanreotide, inteferon alfa, and their combination for therapy of metastatic neuroendocrine gastroenteropancreatic tumors- the international lanreotide and interferon alfa study group," Journal of Clinical Oncology, vol. 21, no. 14, pp. 2689-2696, 2003.

[9] E. T. Janson and K. Oberg, "Long-term management of the carcinoid syndrome. Treatment with octreotide alone and in combination with $\alpha$-interferon," Acta Oncologica, vol. 32, no. 2, pp. 225-229, 1993.

[10] F. Panzuto, M. Di Fonzo, E. Iannicelli et al., "Long-term clinical outcome of somatostatin analogues for treatment of progressive, metastatic, well-differentiated entero-pancreatic endocrine carcinoma," Annals of Oncology, vol. 17, no. 3, pp. 461-466, 2006.

[11] H. Shojamanesh, F. Gibril, L. Adeline et al., "Prospective study of the antitumor efficacy of long-term octreotide treatment in patients with progressive metastatic gastrinoma," Cancer, vol. 94, no. 2, pp. 331-343, 2002. 
[12] I. Buchmann, M. Henze, S. Engelbrecht et al., "Comparison of 68Ga-DOTATOC PET and 111In-DTPAOC (Octreoscan) SPECT in patients with neuroendocrine tumours," European Journal of Nuclear Medicine and Molecular Imaging, vol. 34, no. 10, pp. 1617-1626, 2007.

[13] M. Hofmann, H. Maecke, A. Borner et al., "Biokinetics and imaging with the somatostatin receptor PET radioligand 68Ga-DOTATOC: preliminary data," European Journal of Nuclear Medicine, vol. 28, no. 12, pp. 1751-1757, 2001.

[14] J. Kowalski, M. Henze, J. Schuhmacher, R. M. Helmut, H. Michael, and $\mathrm{H}$. Uwe, "Evaluation of positiron emission tomography imaging using $\left[{ }^{68} \mathrm{Ga}\right]-\mathrm{DOTA}-\mathrm{D}$ Phe(1)-Tyr(3)Octreotide in comparison to $\left[{ }^{111} \mathrm{In}\right]$-DTPAOC SPECT. First results in patients with neuroendocrine tumors," Molecular Imaging \& Biology, vol. 5, no. 1, pp. 42-48, 2003.

[15] D. J. Kwekkeboom, W. Bakker, B. L. Kam et al., "Treatment of patients with gastro-entero-pancreatic (GEP) tumours with the novel radiolabelled somatostatin analogue $\left[{ }^{177} \mathrm{Lu}-\right.$ DOTA $^{0}$, Tyr $^{3}$ ] octreotate," European Journal of Nuclear Medicine and Molecular Imaging, vol. 30, no. 3, pp. 417-422, 2003.

[16] D. J. Kwekkeboom, W. W. de Herder, B. L. Kam et al., "Treatment with the radiolabled somatostatin analog $\left[{ }^{177} \mathrm{Lu}-\right.$ DOTA $^{o}, \mathrm{Tyr}^{3}$ ] octreotate: toxicity, efficacy and survival," Journal of Clinical Oncology, vol. 26, no. 13, pp. 2124-2130, 2008.

[17] A. R. Haug, C. J. Auernhammer, G. P. Schmidt et al., "68GaDOTATATE PET/CT for the early prediction of response to somatostatin receptor-mediated radionuclide therapy in patients with well-differentiated neuroendocrine tumors," Journal of Nuclear Medicine, vol. 51, no. 9, pp. 1349-1356, 2010.

[18] A. Asnacios, F. Courbon, P. Rochaix et al., "Indium-111pentetreotide scintigraphy and somatostatin receptor subtype 2 expression: new prognostic factors for malignant welldifferentiated endocrine tumors," Journal of Clinical Oncology, vol. 26, no. 6, pp. 963-970, 2008.

[19] D. Wild, J. S. Schmitt, M. Ginj et al., "DOTA-NOC, a highaffinity ligand of somatostatin receptor subtypes 2, 3 and 5 for labelling with various radiometals," European Journal of Nuclear Medicine and Molecular Imaging, vol. 30, no. 10, pp. 1338-1347, 2003.

[20] S. Adams, R. Baum, T. Rink, and K. H. Usadel, "Limited value of fluorine-18 fluorodeoxyglucose positron emission tomography for the imaging of neuroendocrine tumours," European Journal of Nuclear Medicine, vol. 25, no. 1, pp. 7983, 1998.

[21] C. Pasquali, D. Rubello, C. Sperti et al., "Neuroendocrine tumor imaging: $\mathrm{can}^{18} \mathrm{~F}$-fluorodeoxyglucose positron emission tomography detect tumors with poor prognosis and aggressive behavior?" World Journal of Surgery, vol. 22, no. 6, pp. 588592, 1998.

[22] S. Koukouraki, L. G. Strauss, V. Georgoulias, M. Eisenhut, U. Haberkorn, and A. Dimitrakopoulou-Strauss, "Comparison of the pharmacokinetics of 68Ga-DOTATOC and [ 18F]FDG in patients with metastatic neuroendocrine tumours scheduled for 90Y-DOTATOC therapy," European Journal of Nuclear Medicine and Molecular Imaging, vol. 33, no. 10, pp. 11151122, 2006.

[23] I. Kayani, J. B. Bomanji, A. Groves et al., "Functional imaging of neuroendocrine tumors with combined PET/CT using 68Ga-DOTATATE (Dota-DPhe1, Tyr3-octreotate) and 18FFDG," Cancer, vol. 112, no. 11, pp. 2447-2455, 2008.

[24] K. P. Zhernosekov, D. V. Filosofov, R. P. Baum et al., "Processing of generator-produced ${ }^{68} \mathrm{Ga}$ for medical application,"
Journal of Nuclear Medicine, vol. 48, no. 10, pp. 1741-1748, 2007.

[25] G. J. Meyer, J. Schuhmacher, W. H. Knapp, and M. Hofmann, "68 Ga-labelled DOTA-derivatised peptide ligands," European Journal of Nuclear Medicine and Molecular Imaging, vol. 31, no. 8, pp. 1097-1104, 2004.

[26] I. Virgolini, V. Ambrosini, J. B. Bomanji et al., "Procedure guidelines for PET/CT tumour imaging with 68Ga-DOTAconjugated peptides: 68Ga-DOTA-TOC, 68Ga-DOTA-NOC, 68Ga-DOTA-TATE," European Journal of Nuclear Medicine and Molecular Imaging, vol. 37, no. 10, pp. 2004-2010, 2010.

[27] R. Boellaard, M. J. O’Doherty, W. A. Weber et al., "FDG PET and PET/CT: EANM procedure guidelines for tumour PET imaging: version 1.0," European Journal of Nuclear Medicine and Molecular Imaging, vol. 37, no. 1, pp. 181-200, 2010.

[28] R. P. Baum, V. Prasad, M. Hommann, and D. Horsch, "Receptor PET/CT imaging of neuroendocrine tumors," Recent Results in Cancer Research. Fortschritte der Krebsforschung, vol. 170, pp. 225-242, 2008.

[29] V. Prasad, V. Ambrosini, M. Hommann, D. Hoersch, S. Fanti, and R. P. Baum, "Detection of unknown primary neuroendocrine tumours (CUP-NET) using 68Ga-DOTANOC receptor PET/CT," European Journal of Nuclear Medicine and Molecular Imaging, vol. 37, no. 1, pp. 67-77, 2010.

[30] A. Rimpler, I. Barth, R. B. Baum, S. Senftleben, and L. Geworski, " $\beta$ radiation exposure of staff during and after therapies with 90Y-labelled substances," Radiation Protection Dosimetry, vol. 131, no. 1, pp. 73-79, 2008.

[31] J. C. Reubi, L. K. Kvols, B. Waser et al., "Detection of somatostatin receptors in surgical and percutaneous needle biopsy samples of carcinoids and islet cell carcinomas," Cancer Research, vol. 50, no. 18, pp. 5969-5977, 1990.

[32] D. J. Kwekkeboom, J. J. Teunissen, W. H. Bakker et al., "Radiolobeled somatostatin analog $\left[{ }^{177} \mathrm{Lu}-\mathrm{DOTA}^{0}, \mathrm{Tyr}^{3}\right]$ octreotate in patients with endocrine gastroenteropancreatic tumors," Journal of Clinical Oncology, vol. 23, no. 12, pp. 2754-2762, 2005.

[33] R. P. Baum and V. Prasad, "Monitoring treatment," in Clinical Nuclear Medicine, G. J. R. Cook, M. N. Maisey, K. E. Britton, and V. Chengazi, Eds., pp. 57-78, Hodder Arnold, London, UK, 4th edition, 2006.

[34] S. Ezziddin, M. Opitz, M. Attassi et al., "Impact of the Ki-67 proliferation index on response to peptide receptor radionuclide therapy," European Journal of Nuclear Medicine and Molecular Imaging, vol. 38, no. 3, pp. 459-466, 2010. 


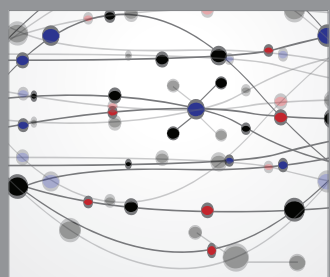

The Scientific World Journal
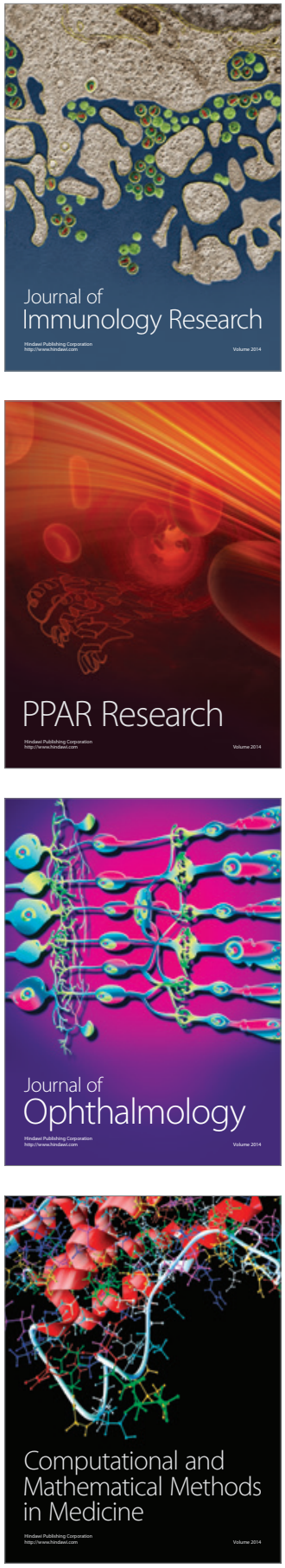

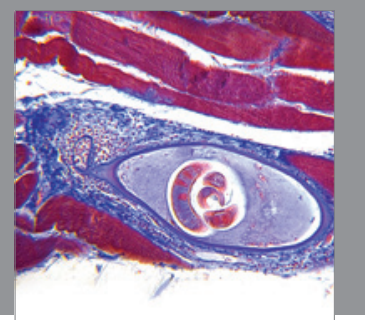

Gastroenterology

Research and Practice
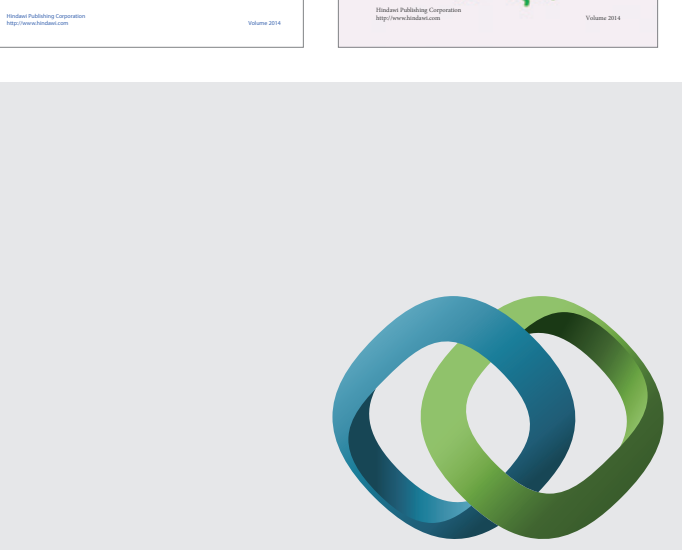

\section{Hindawi}

Submit your manuscripts at

http://www.hindawi.com
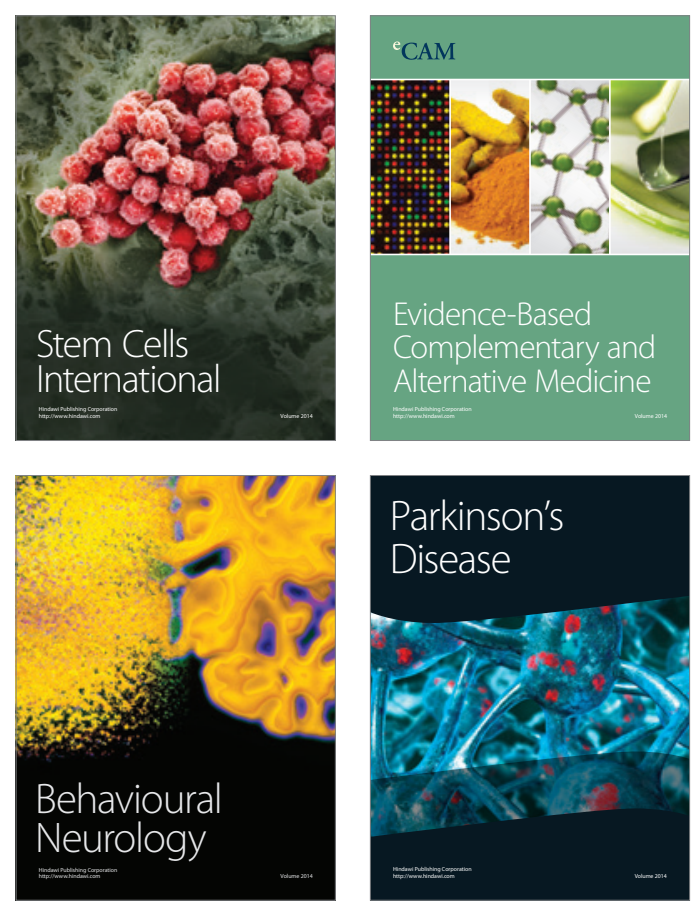

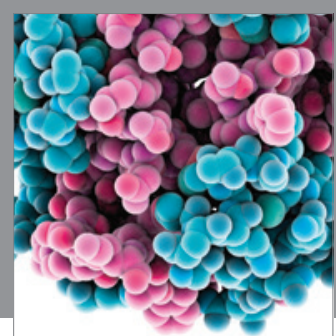

Journal of
Diabetes Research

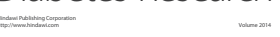

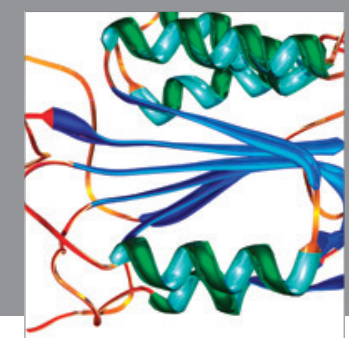

Disease Markers
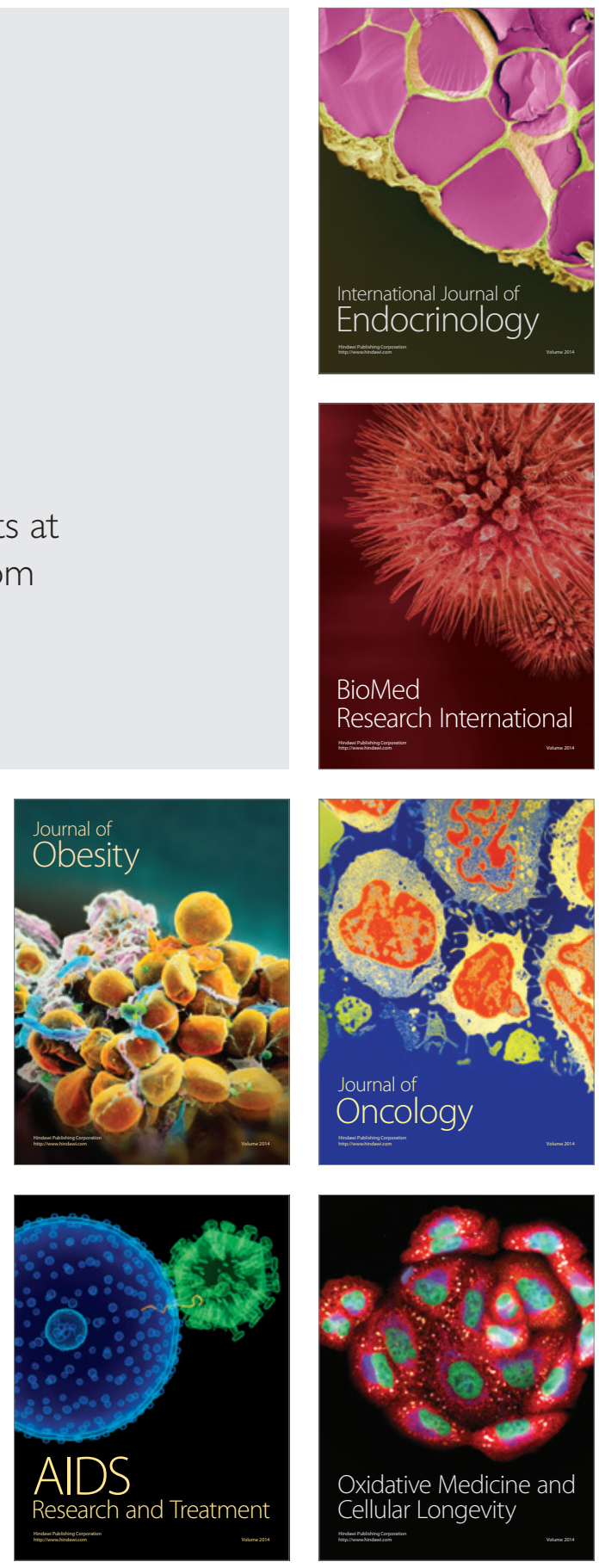